\title{
Absorption of Nitric Oxide in Simulated Flue Gas by Azole-Based Hydrophobic Deep Eutectic Solvents
}

\author{
Ying Sun ${ }^{1}$, Minjie Gao ${ }^{1}$, Long $\operatorname{Lin}^{1}$, Shuhang Ren ${ }^{1}$, Yucui Hou ${ }^{2}$, and Weize $\mathrm{Wu}^{1}$ \\ ${ }^{1}$ Beijing University of Chemical Technology \\ ${ }^{2}$ Taiyuan Normal University
}

September 11, 2020

\begin{abstract}
Functional hydrophobic deep eutectic solvents composed with azoles as hydrogen bond donors, and [N8881]Cl and [N8881]Br as hydrogen bond acceptors were designed and synthesized to absorb low concentrations NO. The good hydrophobicity reduces the cost of operating effectively. Moreover, the capacity of $10 \mathrm{vol} \% \mathrm{NO}$ absorption by Im-[N8881]Cl DES can reach 3.91 mol NO / mol Im at 40 oC. Besides, Im-[N8881]Cl DES can be regenerated, and the absorption capacity of NO by the regenerated Im-[N8881]Cl DES shows no significant loss for at least 5 recycles. FTIR and 1H NMR were used to verify the mechanism. The results indicate that there is chemical interaction between NO and Im-[N8881]Cl DES and the main chemical active sites are $\mathrm{N}$ (2) and N (5) on Im by forming NONOates. This series of hydrophobic, efficient and reversible NO absorbents may provide a new idea for NO capture technique.
\end{abstract}

\section{Hosted file}

my file.docx available at https://authorea.com/users/355803/articles/480562-absorption-ofnitric-oxide-in-simulated-flue-gas-by-azole-based-hydrophobic-deep-eutectic-solvents 